\title{
Perfil de vítimas de queimaduras atendidas num hospital geral de uma capital do nordeste brasileiro 2011 - 2015
}

\author{
Profile of burn victims treated at a general hospital in a capital of the Brazilian Northeast \\ $2011-2015$
}

\begin{abstract}
Perfil de las víctimas de quemaduras atendidas en un hospital general en una capital del noreste de Brasil $2011-2015$
\end{abstract}

Fernando Henrique de Oliveira Santa Maria ${ }^{1}$, Kalleu Leonardo Antão ${ }^{1}$, Marcelo da Silva Pinheiro', Tâmyssa Simões dos Santos ${ }^{2}$, Marcela das Neves Guimarães ${ }^{2 *}$, IIma Ferreira de Oliveira ${ }^{1}$, Alan Blendo Bonfim Correia ${ }^{1}$, Sthylla da Conceição Antão ${ }^{1}$, João Gabriel de Oliveira Santa Maria ${ }^{1}$, Lucyo Wagner Torres de Carvalho'.

\section{RESUMO}

Objetivo: Descrever o perfil epidemiológico de vítimas de queimaduras atendidas em um hospital de uma capital do nordeste brasileiro de 2011 a 2015. Método: Trata-se de um estudo ecológico e retrospectivo, que teve como critérios de inclusão: pacientes atendidos no dito hospital, com diagnostico de queimaduras durante o período de 2011 a 2015. Foram excluídos da amostra prontuários que faziam referência a casos clínicos, fichas sem especificações do atendimento ou aquelas que se mostravam com dados ilegíveis. Resultados: Foram avaliados 1011 prontuários de pessoas atendidas por lesões de queimaduras, destas houve destaque para as crianças de 0 a 9 anos, representando $37,2 \%$. O período de ocorrência mais prevalente foi o vespertino $37,6 \%$, e a região corporal mais lesada foram os membros superiores $25,3 \%$. Realizaram cirurgia $51,1 \%$ e 49,9\% foram tratados clinicamente. Dos 1011 internamentos, 91,4\% evoluíram com alta hospitalar e $3,4 \%$ foram transferidos para outra instituição. A letalidade foi $5,2 \%$. Conclusão: $O$ estudo demonstra as dimensões dos agravos para a população, o sexo masculino e crianças, sendo fatores de risco para queimaduras. Esta pesquisa mostra dados relevantes na epidemiologia desse agravo, o qual pode fomentar a elaboração e implantação de medidas para diminuir a incidência das queimaduras.

Descritores: Serviço Hospitalar de Emergência, Queimaduras, Trauma, Perfil de Saúde, Nordeste.

\begin{abstract}
Objective: To describe the epidemiological profile of burn victims treated in hospital of a capital of the Brazilian Northeast from 2011 to 2015. Method: This is an ecological and retrospective study, whose inclusion criteria were: patients treated at the hospital, diagnosed with burns during the period from 2011 to 2015. Medical records referring to clinical cases, records without specifications were excluded from the sample. or even those with illegible data. Results: A total of 1011 medical records of people treated for burn injuries were evaluated. Of these, the children from zero to nine years old accounted for $37.2 \%$. The most prevalent period of occurrence was evening $37.6 \%$, and the most injured body region were upper limbs $25.3 \%$. They underwent surgery $51.1 \%$ and $49.9 \%$ were clinically treated. Of the 1011 hospitalizations, $91.4 \%$ evolved with hospital discharge and $3.4 \%$ were transferred to another institution. The lethality was $5.2 \%$. Conclusion: The study demonstrates the dimensions of the injuries to the population, the male gender and children, being risk factors for burns. This research shows relevant data on the epidemiology of this disease, which may encourage the development and implementation of measures to reduce the incidence of burns.
\end{abstract}

Descriptors: Emergency Hospital Service, Burns, Trauma, Health Profile, Northeast.

${ }^{1}$ Universidade Estadual de Ciências da Saúde de Alagoas (UNCISAL), Maceió-Alagoas.

${ }^{2}$ Centro Universitário Maurício de Nassau (UNINASSAU), Maceió-Alagoas. *E-mail: marcelaguimaraes.enf@gmail.com

SUBMETIDO EM: 7/2019

ACEITO EM: 8/2019

PUBLICADO EM: $8 / 2019$

REAS/EJCH | Vol.Sup.32 | e1211 | DOI: https://doi.org/10.25248/reas.e1211.2019 Página 1 de 7 


\section{RESUMEN}

Objetivo: Describir el perfil epidemiológico de las víctimas de quemaduras tratadas en un hospital de una capital del noreste de Brasil de 2011 a 2015. Método: Este es un estudio ecológico y retrospectivo, cuyos criterios de inclusión fueron: pacientes tratados en el hospital, diagnosticados con quemaduras durante el período de 2011 a 2015. Fueron excluidos de la muestra fichas médicas que se refieren a casos clínicos, registros sin especificaciones de la asistencia o aquellos con datos ilegibles. Resultados: Se evaluaron un total de 1011 registros de personas tratadas por lesiones de quemaduras, de estas hubo destaque para los niños de 0 a 9 años representando el $37,2 \%$. El período de prevalencia más frecuente fue el $37,6 \%$ vespertino, y la región del cuerpo más lesionada fueron los miembros superiores $25,3 \%$. Se sometieron a cirugía, $51.1 \%$ y $49.9 \%$ fueron tratados clínicamente. De las 1011 hospitalizaciones, el 91,4\% evolucionó con el alta hospitalaria y el 3,4\% se transfirió a otra institución. La letalidad fue del 5,2\%. Conclusión: El estudio demuestra las dimensiones de las lesiones para la población, el sexo masculino y los niños, siendo factores de riesgo de quemaduras. Esta investigación muestra datos relevantes sobre la epidemiología de esta enfermedad, lo que puede alentar el elaboración y la implementación de medidas para reducir la incidencia de quemaduras.

Descriptores: Servicio hospitalário de emergencia, Quemaduras, Trauma, Perfil de salud, Noreste.

\section{INTRODUÇÃO}

A queimadura é definida como uma lesão causada por um agente externo, que pode ser decorrente de trauma térmico, elétrico, químico ou radioativo, levando a uma destruição parcial ou total da pele, causa dor física e em casos potencialmente mais graves, o paciente pode evoluir para óbito. Além do dano tecidual, pode desencadear infecções recorrentes e perda de eletrólitos, o que ocasiona consequentemente mais tempo de permanência no âmbito hospitalar (LIMA MG, 2019).

As causas externas, que são configuradas como acidentes e violências, se apresentam como um grande problema de saúde, ao qual cursa com altas taxas de morbimortalidade (SILVEIRA ES e O'DWYER G, 2017).

As queimaduras são, em todo mundo consideradas como um problema de saúde pública global. Na média anual são notificados cerca de 180.000 mortes. E o que chama atenção, que estes casos ocorrem em sua maioria em países de baixa e média renda, havendo destaque para as regiões da África e Sudeste Asiático. Sua importância decorre não apenas da grande incidência, mas, sobretudo, da capacidade de produzir sequelas, as incapacidades variam de temporária a permanente, sendo incidentes em $17 \%$ das crianças nos países da Bangladesh, Colômbia, Egito e Paquistão (WHO, 2018).

No Brasil, estima-se que anualmente ocorra 1 milhão de pessoas com traumas por queimaduras (SILVEIRA ES e O'DWYER G, 2017).

E estes indivíduos demandam do atendimento dos serviços hospitalares, o que gera um custo anual de 63 milhões de reais (SANTOS GP, et al., 2017).

No ano de 2016 foi registrado 22.719 internações, e neste cenário, o Nordeste ocupa a posição de segundo lugar com 7.071 hospitalizações. Esta afecção resulta em danos aos sistemas respiratório, imunológico, cardiovascular e renal, geralmente acompanhado de um quadro de hipovolemia, hipotensão, taquicardia e choque (MOLA R, et al., 2018).

Além do mais, implica diretamente na qualidade de vida e condições de trabalho das pessoas acometidas (RYAN CM, et al., 2015), e afetam grupos vulneráveis como as crianças, os idosos e indivíduos com necessidades especiais, o que pode gerar em traumas de cunho emocional, psicológico e social (DUTRA JPS, et al., 2017).

Desse modo, o presente estudo teve como objetivo descrever o perfil epidemiológico de vítimas de queimaduras atendidas em um hospital de uma capital do nordeste brasileiro de 2011 a 2015. Tendo em vista a necessidade de desenvolvimento de ações de prevenção bem como estratégias para a fomentação de política publicas volta para a prevenção do agravo. 


\section{MÉTODOS}

Trata-se de um estudo ecológico e retrospectivo. O estudo ocorreu em um hospital, que recebe pacientes das diversas regiões do Estado de Alagoas. É o único hospital que possui unidade em Alagoas especializada no tratamento de queimaduras.

A coleta e análise dos dados ocorreu por intermédio de prontuários dos pacientes internados. Os critérios de inclusão foram: pacientes atendidos no dito hospital, com diagnostico de queimaduras durante o período de 2011 a 2015. Foram excluídos da amostra prontuários que faziam referência a casos clínicos, fichas sem especificação do atendimento ou mesmo aquelas que se mostravam com dados ilegíveis.

Com a triagem dos prontuários atendendo apenas ao critério de internamento por queimaduras, as informações foram sistematizadas e, os dados, extraídos e inseridos em formulário próprio de coleta. As variáveis categorizadas para este estudo, foram: ano, sexo, faixa etária, mês e horário de maior ocorrência, meio de condução, parte do corpo atingida, tratamento e evolução.

Logo, estes foram organizados e armazenados em planilha eletrônica Microsoft Excel®. Desse modo, buscou-se interpretar os resultados utilizando o teste Qui-quadrado, que possibilitou verificar as prevalências e/ou associações entre as variáveis, dependentes ou independentes. Assim, ressalta-se que o programa utilizado foi o Bioestat $5.0 \mathrm{com}$ alfa igual a 0,05.

Esta pesquisa foi aprovada pelo Comitê de Ética em Pesquisa (CEP) da Universidade Estadual de Ciências da Saúde de Alagoas (UNCISAL), através do Certificado de Apresentação para Apreciação Ética (CAAE) no 1.572.270, estando este de acordo com o que preconiza a Resolução 466/2012, do Conselho Nacional de Saúde com pesquisas envolvendo seres humanos.

\section{RESULTADOS}

No período de 01 de janeiro de 2011 a 31 de dezembro de 2015 ocorreram 1011 internações por queimaduras. O primeiro passo foi avaliar a caracterização anual dos atendimentos. Observou-se que durante o período avaliado que houve maior ocorrência de internações por queimaduras no ano de 2011 com 228 internamentos. Houve um decréscimo nos anos seguintes, e a partir de 2014 voltou a aumentar o número de casos, com 181, e 2015 com 225 internamentos.

$\mathrm{Na}$ avaliação os pacientes do sexo masculino foram os mais acometidos entre os anos de 2011 e 2015 ,

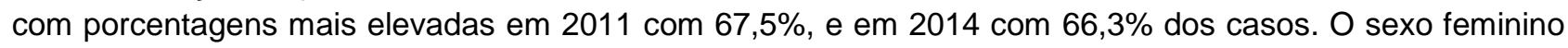
apresentou um crescimento no ano de 2015 com 87 (38,7\%) internações (Tabela 1).

Tabela 1 - Caracterização anual dos internamentos por queimaduras, segundo sexo. Maceió, Alagoas, Brasil, 2011-2015.

\begin{tabular}{ccccccc}
\hline \multicolumn{1}{c}{ M } & \% & F & $\%$ & Total & P \\
\hline Anos & & & & & & \\
\hline $\mathbf{2 0 1 1}$ & 154 & 67,5 & 74 & 32,5 & 228 & \\
$\mathbf{2 0 1 2}$ & 129 & 64,5 & 71 & 35,5 & 200 & 0.6939 \\
$\mathbf{2 0 1 3}$ & 117 & 66,1 & 60 & 33,9 & 177 & \\
$\mathbf{2 0 1 4}$ & 120 & 66,3 & 61 & 33,7 & 181 & \\
$\mathbf{2 0 1 5}$ & 138 & 61,3 & 87 & 38,7 & 225 & \\
\hline
\end{tabular}

Fonte: Serviço de Arquivamento Médico (SAME) - HGE.

Descrito o perfil dos atendimentos, a partir da descrição verificou-se que houve variação significativa entre meses $(p=0,04)$ e meios de condução $(p<0,001)$. Notou-se que os indivíduos de $0-9$ anos foram os mais acometidos (37,2\%), seguido das pessoas com 20-39 anos (26,6\%) e em terceiro lugar, a faixa etária 40-59 $(15,4 \%)$. 
Observou-se que a maior parte das ocorrências por queimaduras ocorrem entre o período da tarde e da noite com cerca de $70,0 \%$ dos casos e menor no período da madrugada com $5,1 \%$. Em relação aos meses do ano no período em análise, a maioria das internações por queimaduras ocorreram em janeiro, março, junho e dezembro, e menor ocorrência nos meses de abril e novembro. O meio de condução mais utilizado foi o particular (54,6\%) em todas as internações estudadas no período, e em segundo outras ambulâncias (30,6\%), seguido do Serviço de Atendimento Móvel de Urgência - Unidade de Suporte Básico (SAMU UBS) (11,5\%), bombeiros (2,7\%) e do Serviço de Atendimento Móvel de Urgência - Unidade de Suporte Avançado (SAMU USA) $(0,7 \%)$ (Tabela 2$)$.

Tabela 2 - Caracterização dos internamentos por queimaduras, segundo agravo. Maceió, Alagoas, Brasil, 2011-2015.

\begin{tabular}{|c|c|c|c|c|c|c|c|}
\hline & \multicolumn{2}{|c|}{ MASCULINO } & \multicolumn{2}{|c|}{ FEMININO } & \multicolumn{2}{|c|}{ TOTAL } & \multirow[t]{2}{*}{$\mathbf{P}$} \\
\hline & $\mathrm{N}$ & $\%$ & $\mathrm{~N}$ & $\%$ & $\mathrm{~N}$ & $\%$ & \\
\hline \multicolumn{8}{|l|}{ Faixa etária } \\
\hline 0 a 9 & 248 & 37,7 & 128 & 36,3 & 376 & 37,2 & \\
\hline 10 a 19 & 95 & 14,4 & 46 & 13,0 & 141 & 13,9 & 0.5322 \\
\hline 20 a 39 & 178 & 27,1 & 91 & 25,8 & 269 & 26,6 & \\
\hline 40 a 59 & 92 & 14,0 & 64 & 18,1 & 156 & 15,4 & \\
\hline 60 e mais & 45 & 6,8 & 24 & 6,8 & 69 & 6,8 & \\
\hline \multicolumn{8}{|l|}{ Meses } \\
\hline Janeiro & 64 & 9,7 & 36 & 10,2 & 100 & 9,9 & \\
\hline Fevereiro & 50 & 7,6 & 29 & 8,2 & 79 & 7,8 & \\
\hline Março & 65 & 9,9 & 35 & 9,9 & 100 & 9,9 & \\
\hline Abril & 44 & 6,7 & 20 & 5,7 & 64 & 6,3 & \\
\hline Maio & 56 & 8,5 & 21 & 5,9 & 77 & 7,6 & \\
\hline Junho & 80 & 12,2 & 20 & 5,7 & 100 & 9,9 & 0.0418 \\
\hline Julho & 51 & 7,8 & 41 & 11,6 & 92 & 9,1 & \\
\hline Agosto & 45 & 6,8 & 30 & 8,5 & 75 & 7,4 & \\
\hline Setembro & 62 & 9,4 & 28 & 7,9 & 90 & 8,9 & \\
\hline Outubro & 44 & 6,7 & 32 & 9,1 & 76 & 7,5 & \\
\hline Novembro & 36 & 5,5 & 24 & 6,8 & 60 & 5,9 & \\
\hline Dezembro & 61 & 9,3 & 37 & 10,5 & 98 & 9,7 & \\
\hline \multicolumn{8}{|l|}{ Horários } \\
\hline Manhã & 156 & 23,7 & 96 & 27,2 & 252 & 24,9 & \\
\hline Tarde & 241 & 36,6 & 139 & 39,4 & 380 & 37,6 & 0.2178 \\
\hline Noite & 223 & 33,9 & 104 & 29,5 & 327 & 32,3 & \\
\hline Madrugada & 38 & 5,8 & 14 & 4,0 & 52 & 5,1 & \\
\hline \multicolumn{8}{|l|}{ Meio de Condução } \\
\hline SAMU USA $^{*}$ & 6 & 0,9 & 1 & 0,3 & 7 & 0,7 & \\
\hline SAMU USB ${ }^{* *}$ & 75 & 11,4 & 41 & 11,6 & 116 & 11,5 & \\
\hline Bombeiros & 20 & 3,0 & 7 & 2,0 & 27 & 2,7 & $<0.0001$ \\
\hline Particular & 349 & 53,0 & 203 & 57,5 & 552 & 54,6 & \\
\hline Outros & 208 & 31,6 & 101 & 28,6 & 309 & 30,6 & \\
\hline
\end{tabular}

Fonte: Serviço de Arquivamento Médico (SAME) - HGE.

Nota: * Serviço de Atendimento Móvel de Urgência - Unidade de Suporte Avançado.

** Serviço de Atendimento Móvel de Urgência - Unidade de Suporte Básico. 
Sistematiza-se a frequência das regiões corporais atingidas por queimaduras. Ao analisar a área mais lesada no período do estudo, verificou-se que em ambos os sexos a área mais acometida em pacientes queimados foram os membros superiores $(25,3 \%)$, seguido do membro inferior $(21,4 \%)$ e tórax $(17,9 \%)$. Entre as regiões menos acometidas, estão as genitálias $(2,9 \%)$ e o dorso $(5,3 \%)$. Não houve diferença entre os sexos e áreas lesadas (Tabela 3).

Tabela 3 - Distribuição dos internamentos por queimaduras, segundo frequência de região corporal atingida. Maceió, Alagoas, Brasil, 2011-2015.

\begin{tabular}{crrrrrr}
\hline Parte do Corpo Atingida & M & \multicolumn{1}{c}{$\%$} & \multicolumn{1}{c}{ F } & \multicolumn{1}{c}{$\%$} & \multicolumn{1}{c}{ TOTAL } & \multicolumn{1}{c}{$\%$} \\
\hline Tórax & 237 & 17,5 & 124 & 18,8 & 361 & 17,9 \\
\hline Abdome & 135 & 10,0 & 67 & 10,1 & 202 & 10,0 \\
\hline Dorso & 73 & 5,4 & 34 & 5,1 & 107 & 5,3 \\
\hline Cabeça/Pescoço & 234 & 17,3 & 111 & 16,8 & 345 & 17,1 \\
\hline Membros Superiores & 343 & 25,4 & 166 & 25,1 & 509 & 25,3 \\
\hline Membros Inferiores & 288 & 21,3 & 142 & 21,5 & 430 & 21,4 \\
\hline Genitálias & 42 & 3,1 & 17 & 2,6 & 59 & 2,9 \\
\hline
\end{tabular}

Fonte: Serviço de Arquivamento Médico (SAME) - HGE.

Descrito o perfil dos desfechos dos casos, a partir da descrição verificou-se que houve variação significativa entre tipo de tratamento $(p<0,05)$ entre o trauma em questão. Ao analisar evolução para pacientes queimados foi identificado que não existiu um tipo de tratamento mais predominante, pois, aproximadamente $49 \%$ dos casos foram para tratamentos clínicos e aproximadamente $51 \%$ dos agravos foram para cirúrgico. Ao analisar o tipo de tratamento para pacientes queimados verificou-se que em $91,4 \%$ dos pacientes tiveram alta, $5,2 \%$ foram a óbito e 3,4\% foram transferidos (Tabela 4 ).

Tabela 4. Distribuição dos internamentos por queimadura, segundo desfechos dos casos. Maceió, Alagoas, Brasil, 2011-2015.

\begin{tabular}{|c|c|c|c|c|c|c|c|}
\hline & \multicolumn{2}{|c|}{$\mathbf{M}$} & \multicolumn{2}{|c|}{$F$} & \multicolumn{2}{|c|}{ TOTAL } & \multirow[t]{2}{*}{$\mathbf{P}$} \\
\hline & $\mathrm{N}$ & $\%$ & $\mathrm{~N}$ & $\%$ & $\mathrm{~N}$ & $\%$ & \\
\hline \multicolumn{8}{|l|}{ Tratamento } \\
\hline Clínico & 304 & 46,2 & 190 & 53,8 & 494 & 48,9 & \\
\hline Cirúrgico & 354 & 53,8 & 163 & 46,2 & 517 & 51,1 & 0.0247 \\
\hline \multicolumn{8}{|l|}{ Evolução } \\
\hline Alta & 596 & 90,6 & 328 & 92,9 & 924 & 91,4 & \\
\hline Transferência & 24 & 3,6 & 10 & 2,8 & 34 & 3,4 & 0.4468 \\
\hline Óbito & 38 & 5,8 & 15 & 4,2 & 53 & 5,2 & \\
\hline
\end{tabular}

Fonte: Serviço de Arquivamento Médico (SAME) - HGE.

\section{DISCUSSÃO}

Dependendo da gravidade, a queimadura pode trazer inúmeras sequelas e os fatores associados a ela podem contribuir significativamente no desfecho da evolução do paciente (MOLA R, et al., 2018). A faixa etária apresentou um marcador importante para esta discussão, da mesma forma o sexo, sendo mais prevalente em indivíduos do sexo masculino e com idade entre 0 e 9 anos. A partir de um levantamento bibliográfico, essa realidade foi evidenciada em outros estudos (JUNIOR RAS, et al., 2016; LEITE VHO, et al., 2016; RYAN CM, et al., 2015; RICCI FPFM, et al., 2015; SANCHES PHE, et al., 2016; SILVEIRA ES e O'DWYER, 2017). 
Para se ter uma dimensão, no estudo de Junior RAS, et al. (2016), 51,1\% dos pacientes estavam entre a faixa etária de 0 a 12 anos. O risco maior para essa faixa etária pode estar associado a imaturidade tanto cognitiva como física, além do aspecto da curiosidade e fase de descobertas (MESCHIAL WC, et al., 2016). Em estudo realizado em Sergipe, a faixa etária mais acometida era entre 18 e 60 anos, com 82,8\%. Em outro estudo, a faixa etária entre 18 a 30 anos foi a maioria (LEITE VHO, et al., 2016), o que comprova uma variação considerável de pacientes atendidos por queimaduras no que diz respeito ao sexo e a idade.

Ao comparar esses achados com estudos internacionais, um realizado em Bagdá (Iraque) e outro realizado em Daca (Bangladesh), o primeiro confirma a predominância de pacientes do sexo masculino, principalmente jovens adultos e crianças. Dentre as principais causas de queimaduras destacam as chamas e escaldões (LAMI FH e NASSER RKA, 2019). Do mesmo modo, o segundo reforça que os grupos de maior risco era o de homens adultos jovens na faixa de 30 anos e de nível socioeconômico mais baixo e de crianças menores de oito anos, considerando as principais causas a chama, instalações elétricas e escaldaduras (BAILEY ME, et al., 2019).

Francisconi MHG, et al. (2016) levanta a hipótese de esses resultados podem ter uma relação direta com as diferenças comportamentais de cada sexo, bem como aos fatores culturais, que dispõe maior liberdade para os indivíduos do sexo masculino e impõe maior vigilância às meninas.

Em se tratando do horário de atendimento, demostrou que para as queimaduras foi mais significativo o turno vespertino com 380 casos (37,6\%). Para Moraes OS, et al. (2014) essa evidencia propõe uma análise relevante, com o maior número de ocorrências no período vespertino, sendo este considerado horário de diversão, favorece o envolvimento e/ou a exposição em situações de violência que levem à queimadura (MORAES PS, et al., 2014). Nesta avaliação, o período noturno também traz um sinal de alerta $(32,3 \%)$.

Por outro lado, um estudo realizado em Macapá-AP aborda que se deve levar em consideração os perigos que estão presentes no dia-a-dia dos indivíduos, principalmente das crianças, a cozinha está entre os ambientes mais perigosos de uma residência. Uma forma de prevenir acidentes por queimaduras entre crianças consiste na participação da família e da escola como agentes formadores de comportamentos preventivos (CORREA RC, 2016).

Quanto a distribuição mensal dos internamentos durante os anos cursou com variação de padrão $(p<0,0418)$. Para as queimaduras janeiro, março e junho, ambos meses com 100 casos $(9,9 \%)$. Uma das causas para o número de queimaduras terem endossado no mês de junho, por exemplo, pode estar relacionado com as festas juninas, comemoradas com grande intensidade na região do Nordeste brasileiro, as brincadeiras de pular fogueiras e as falhas decorrentes do manuseio de fogos de artifício colocam os adultos e, principalmente as crianças, em risco elevado para acidentes por queimaduras (CORREA RC, 2016). Em se tratando de estudo realizado em Londrina, os agravos tiveram seus números aumentados no período de outono e inverno (MORAES, et al., 2014).

No que concerne ao meio de condução ao hospital, foi observado, no agravo em questão, que a locomoção de modo particular predominou nas queimaduras $542(53,6 \%)$, sendo seguido por outras, como ambulâncias de unidades de saúde do interior do Estado, 309 (30,6\%).

No aspecto relacionado ao número de lesões provocadas por queimaduras, evidenciou-se que a maioria das vítimas apresentou lesões em membros superiores (25,3\%). Em outros estudos, os membros superiores foram os mais acometidos (SANCHES PHS, et al., 2016; MORAES OS, et al., 2014; COSTA ACSM, et al., 2016), divergindo do estudo de Leite VHO et al. (2016), a qual a face representou (48,4\%) dos atendidos. No quesito que avaliava as condutas, observou-se que os procedimentos cirúrgicos predominaram nas queimaduras 517 (51,1\%). Em relação a evolução dos casos estudados, observou-se para este agravo, as altas predominaram com 924 casos $(91,4 \%)$. A taxa de letalidade nas queimaduras foi $5,2 \%$. Verificou-se em uma dada pesquisa que a mortalidade foi 2,31\% (JUNIOR RAS, et al., 2016), já em um estudo paulista 5,6\% (DIAS LDF, et al., 2015). O que demostra um percentual reduzidos em diversas regiões do país, no entanto, ainda é um dado alarmante, por se tratar de uma lesão que pode ser prevenida. 
As limitações do estudo foram, principalmente, de ordem física, haja visto que os prontuários eram armazenados em caixas de papel e sem nenhuma subdivisão. Este fator dificultava, tanto o manuseio, como a interpretação dos dados neles contidos. Outros pontos que merecem ser destacados, são os casos das letras ilegíveis ou incompreensíveis, assim como, a ausência de dados relevantes que foram subnotificados e poderiam ajudar a delimitar outras vertentes importantes.

\section{CONCLUSÃO}

Dentro desta pesquisa tornou-se possível fazer o levantamento de alguns aspectos relevantes sobre o perfil epidemiológico das queimaduras. O sexo masculino esteve mais prevalente nas queimaduras. Observou-se também que a maior prevalência nas pacientes vítimas de queimaduras eram crianças. Esta pesquisa mostra dados importantes e relevantes na epidemiologia dos respectivos agravos, os quais podem fomentar a elaboração e implantação de medidas para prevenir e diminuir a incidência dos agravos em questão, considerando-se o grande impacto destes agravos na sociedade.

\section{REFERÊNCIAS}

1. BAILEY ME, et al. Epidemiology and outcomes of burn injuries at a tertiary burn care center in Bangladesh. Burns, 2019; 45(4): 957-963.

2. BRASIL. Ministério da Saúde. Secretaria de Atenção à Saúde. Departamento de Atenção Especializada. Cartilha para tratamento de emergência das queimaduras/Ministério da Saúde, Secretaria de Atenção à Saúde, Departamento de Atenção Especializada. Brasília: Editora do Ministério da Saúde, 2012; 20p. (Série F. Comunicação e Educação em Saúde).

3. COSTA ACSM, et al. Amplitude de movimento e sua interferência na capacidade funcional de pacientes com sequelas de queimaduras. Revista Brasileira de Queimaduras, 2016; 15(4): 261-6.

4. CORREA RC. Incidência de pacientes queimados atendidos no Hospital de Emergência na cidade de Macapá-AP, durante o ano de 2014. Estação Científica (UNIFAP), 2016; 6(1): 53-61.

5. DIAS LDF, et al. Unidade de Tratamento de Queimaduras da Universidade Federal de São Paulo: estudo epidemiológico. Revista Brasileira de Cirurgia Plástica, 2015; 30(1): 86-92.

6. DUTRA JPS, et al. Estudo clínico-epidemiológico de pacientes queimados internados em uma unidade de terapia intensiva em Goiás. Revista Brasileira de Queimaduras, 2017; 16(2): 87-93.

7. FRANCISCONI MHG, et al. Perfil epidemiológico das crianças com queimaduras hospitalizadas em um Centro de Tratamento de Queimados. Revista Brasileira de Queimaduras, 2016; 15(3): 131-6.

8. JUNIOR RAS, et al. Perfil epidemiológico dos pacientes queimados no Hospital de Urgências de Sergipe. Revista Brasileira de Queimaduras, 2016; 15(4): 251-5.

9. LAMI FH, NASSER RKA. Epidemiological characteristics of burn injuries in Iraq: a burn hospital-based study. Burns, 2019; 45(2): 479-483.

10. LEÃO CEG, et al. Epidemiologia das queimaduras no estado de Minas Gerais. Revista Brasileira de Cirurgia Plástica, 2011; 26(4): 573-577.

11. LEITE VHO, et al. Análise dos acidentes por queimadura com álcool líquido em Unidade de Tratamento de Queimados em Sergipe. Revista Brasileira de Queimaduras, 2016; 15(4): 235-239.

12. LIMA MG, et al. Análise do perfil dos casos de queimaduras em Sergipe nos anos de 2013-2018. Brazilian Journal of Health Review, 2019; 2(3): 1555-1561.

13. MESCHIAL WC, et al. Fatores de risco e medidas de prevenção das queimaduras infantis: revisão integrativa da literatura. Revista Brasileira de Queimaduras, 2016; 15(4): 267-273.

14. MOLA R, et al. Características e complicações associadas às queimaduras de pacientes em unidade de queimados. Revista Brasileira de Queimaduras, 2018; 7(1): 1-6.

15. MORAES PS, et al. Perfil das internações de crianças em um centro de tratamento para queimados. Revista Eletrônica de Enfermagem, 2014; 16(3): 598-603.

16. RICCI FPFM, et al. Perfil epidemiológico dos pacientes com queimadura em membros superiores atendidos em uma Unidade de Queimados terciária. Revista Brasileira de Queimaduras, 2015; 14(1): 10-13.

17. RYAN CM, et al. Multicenter Burn Outcome Group. Recovery trajectories after burn injury in young adults: does burn size matter? Journal of Burn Care \& Research, 2015; 36(1): 118-29.

18. SANCHES PHS, et al. Perfil epidemiológico de crianças atendidas em uma Unidade de Tratamento de Queimados no interior de São Paulo. Revista Brasileira de Queimaduras, 2016; 15(4): 246-50.

19. SANTOS GP, et al. Perfil epidemiológico do adulto internado em um centro de referência em tratamento de queimaduras. Revista Brasileira de Queimaduras, 2017; 16(2): 81-6.

20. SILVEIRA ES, O'DWYER G. Centro de Trauma: modelo alternativo de atendimento às causas externas no Estado do Rio de Janeiro. Saúde debate [online], 2017; 41(112): 243-254.

21. WORLD HEALTH ORGANIZATION (WHO). Burn prevention: sucess stories and lessons learned [Internet]. Geneva; 2011.

22. WORLD HEALTH ORGANIZATION (WHO). Burns [Internet], 2018. 